Supporting Information

\title{
Systematic study of Al impurity for NCM622 cathode materials
}

\author{
Ruihan Zhang ${ }^{1}$, Yadong Zheng ${ }^{1}$, Zeyi Yao ${ }^{1}$, Panawan Vanaphuti ${ }^{1}$, Xiaotu $\mathrm{Ma}^{1}$, Sungyool Bong ${ }^{1}$, \\ Mengyuan Chen ${ }^{1}$, Yangtao Liu ${ }^{1}$, Feng Cheng ${ }^{2}$, Zhenzhen Yang ${ }^{3}$, Yan Wang ${ }^{1 *}$ \\ ${ }^{1}$ Department of Mechanical Engineering, Worcester Polytechnic Institute, 100 Institute Road, \\ Worcester, MA 01609 \\ 2 Department of Chemical Engineering, Worcester Polytechnic Institute, 100 Institute Road, \\ Worcester, MA 01609 \\ ${ }^{3}$ Argonne National Laboratory, 9700 S Cass Ave, Lemont, IL 60439
}

*Correspondence: yanwang@wpi.edu (Y.W.), Tel.: +1-508-831-5453

General Content of Supporting Information (SI) file:

Total number of SI pages: 7 pages

Total number of figures: 9 figures

Total number of tables: 2 tables 


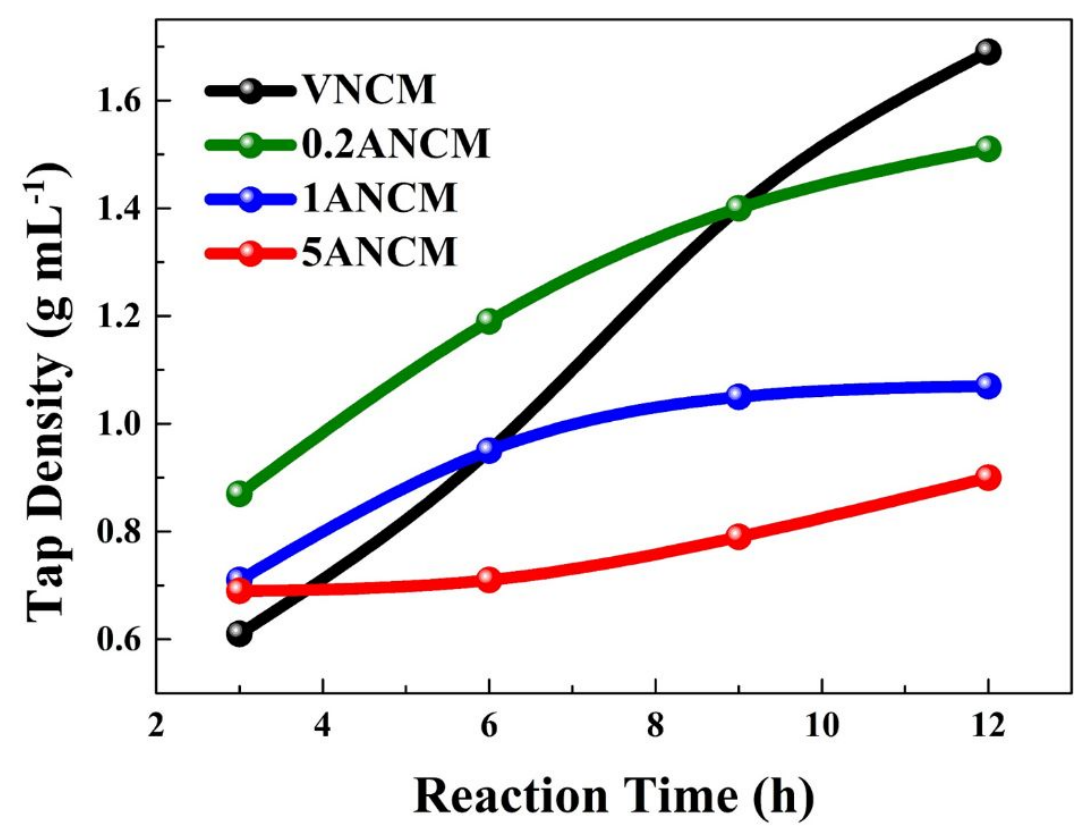

Figure S1. Tap density changes for the prepared precursors at different reaction times.

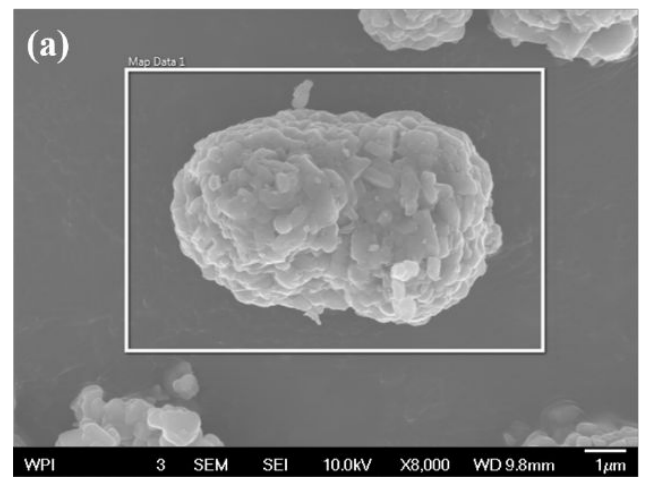

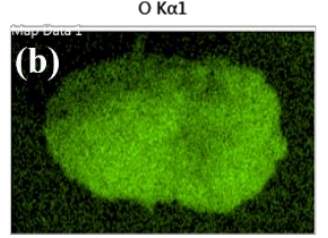

$\mathrm{Mn} \mathrm{K} \alpha 1$

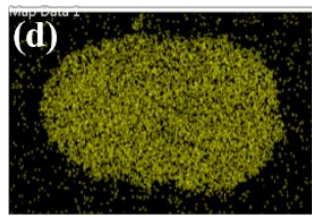

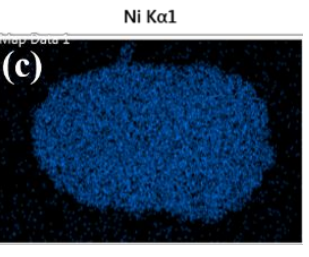

Co K $\alpha 1$

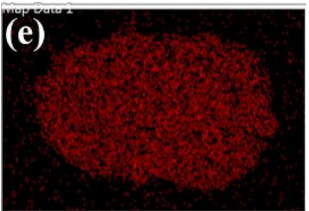

Figure S2. Material characterization of the VNCM cathode particles. (a) The SEM images and EDX mapping results of (b) $\mathrm{O},(\mathrm{c}) \mathrm{Ni}$, (d) $\mathrm{Mn}$ and (e) Co elements distribution. 

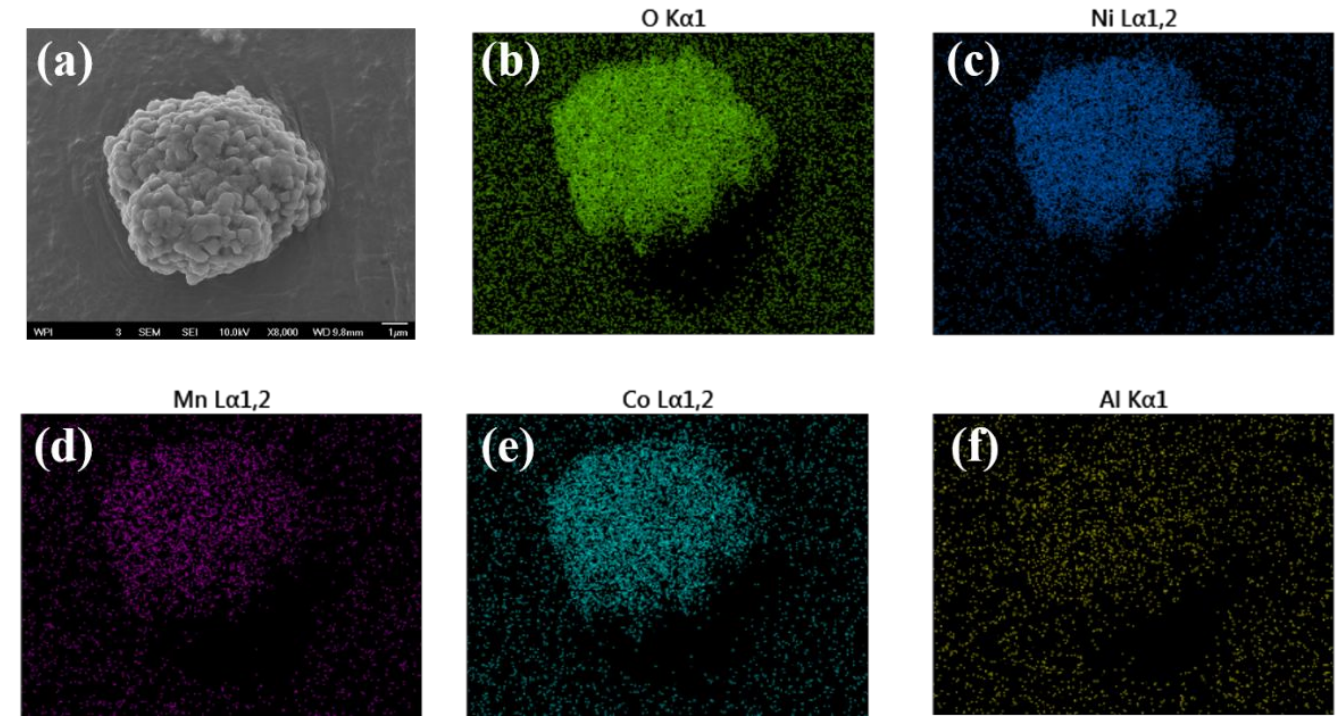

Figure S3. Material characterization of the $0.2 \mathrm{ANCM}$ cathode particles. (a) The SEM images and EDX mapping results of (b) $\mathrm{O}$, (c) $\mathrm{Ni}$, (d) Mn, (e) Co and (f) Al elements distribution.
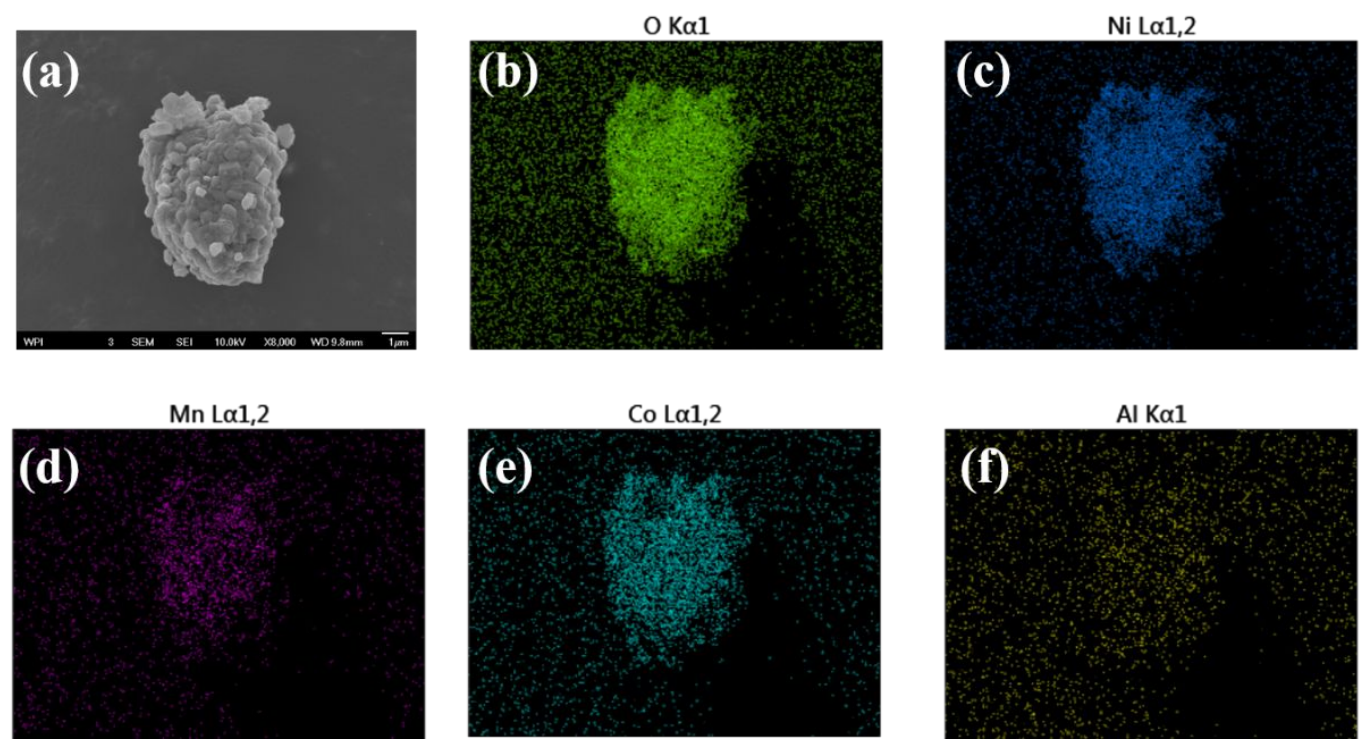

Figure S4. Material characterization of the 1ANCM cathode particles. (a) The SEM images and EDX mapping results of (b) $\mathrm{O}$, (c) $\mathrm{Ni}$, (d) Mn, (e) Co and (f) Al elements distribution. 


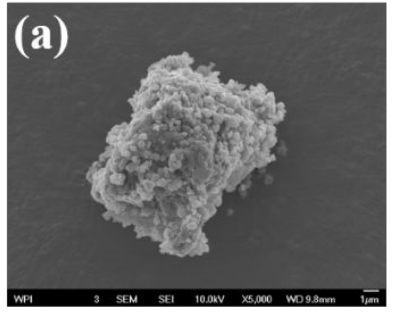

Mn $L \alpha 1,2$

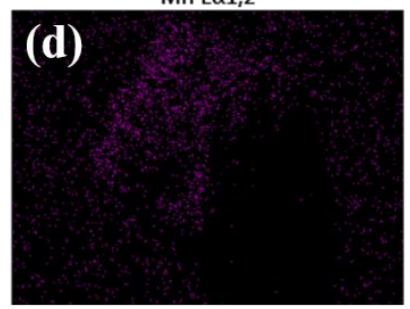

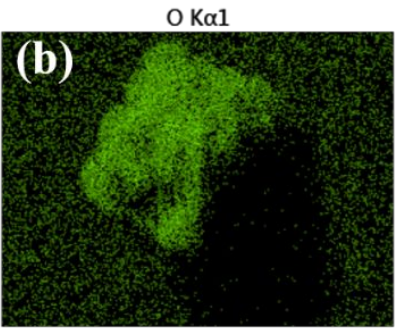

Co $L \alpha 1,2$

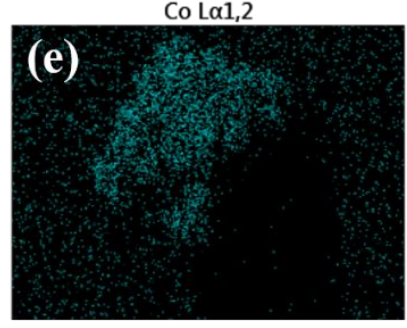

Ni $L \alpha 1,2$

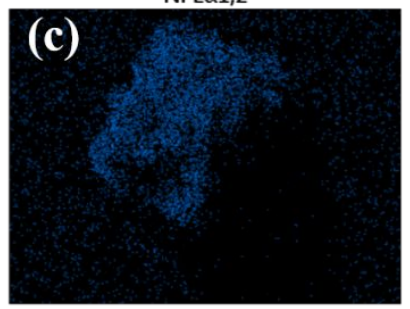

Al K $\alpha 1$

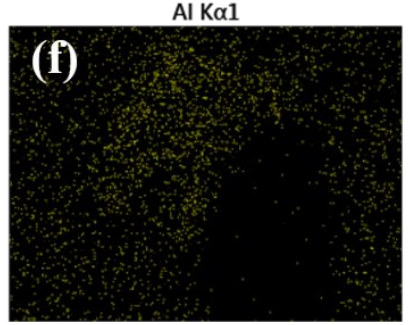

Figure S5. Material characterization of the 5ANCM cathode particles. (a) The SEM images and EDX mapping results of (b) $\mathrm{O}$, (c) $\mathrm{Ni}$, (d) $\mathrm{Mn}$, (e) Co and (f) Al elements distribution.
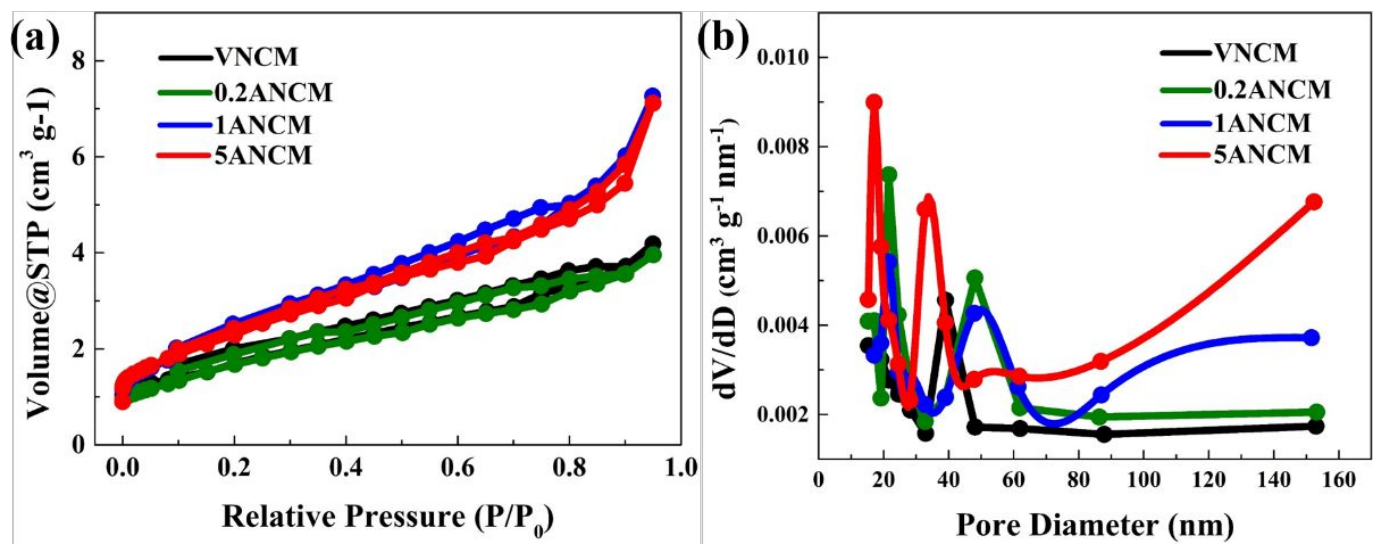

Figure S6. (a) Nitrogen adsorption-desorption isotherm of the NCM622 cathode samples; (b) Pore size distribution of the synthesized cathode samples. 


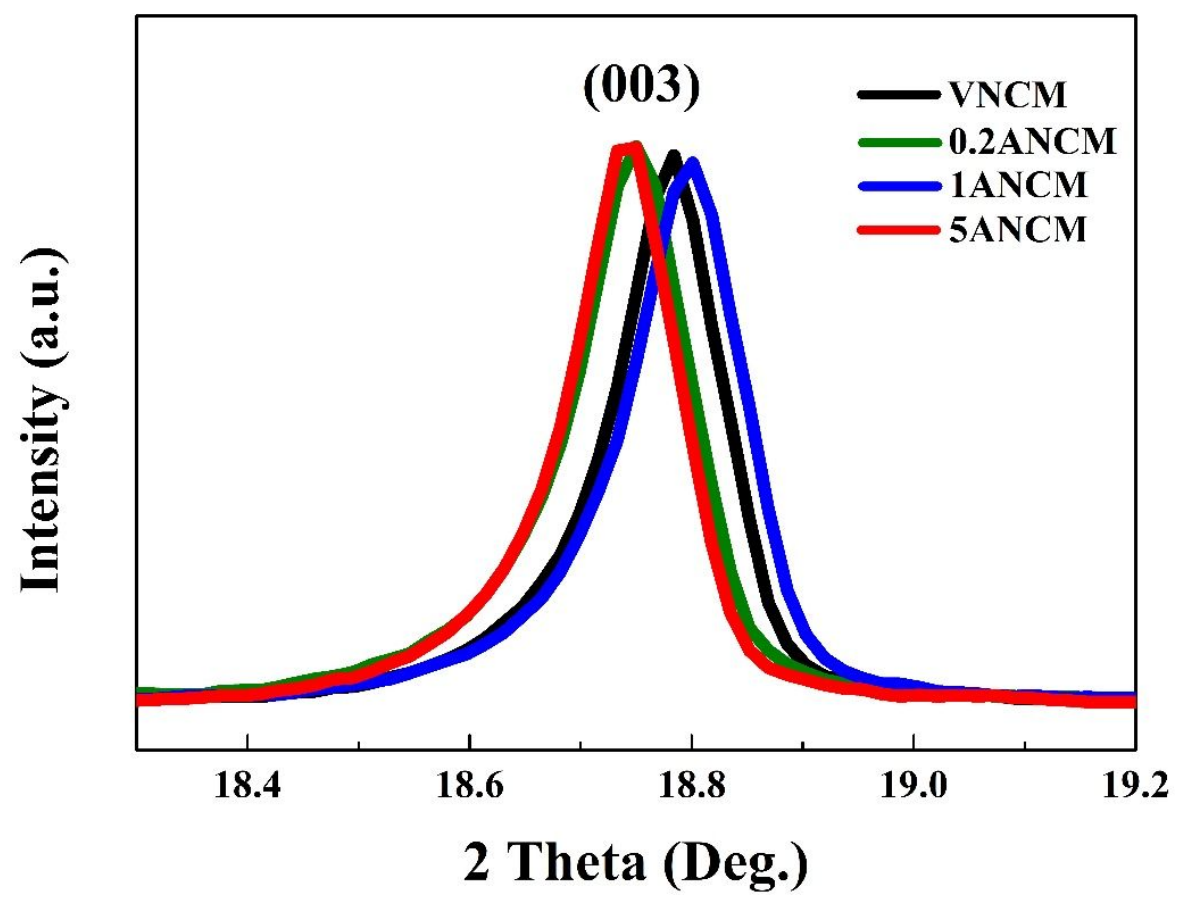

Fig. S7. The detailed display of the (003) peak of VNCM, 0.2ANCM, 1ANCM and 5ANCM cathode materials.
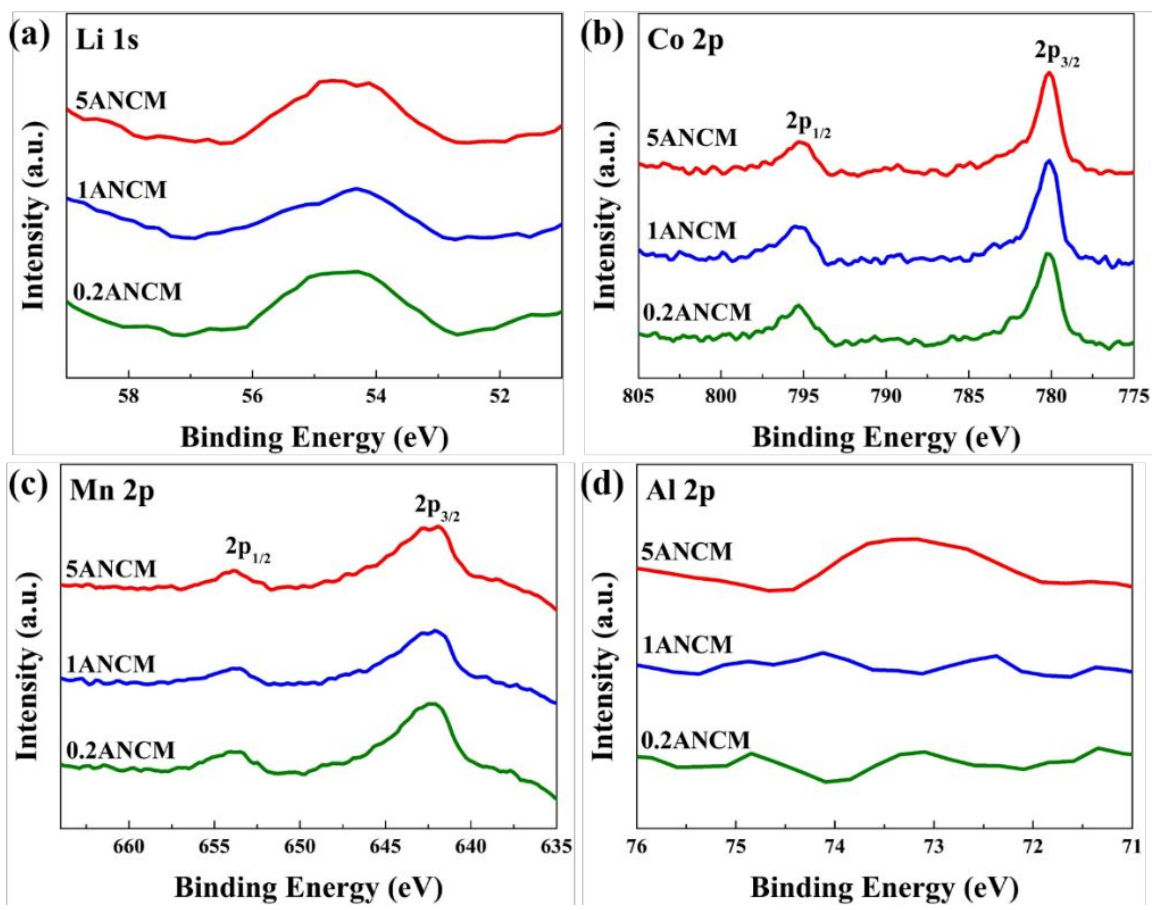
Figure S8. The XPS spectra of (a) Li 1s, (b) Co 2p, (c) Mn 2p and (d) Al 2p for the prepared 0.2ANCM, 1ANCM and 5ANCM cathode samples.
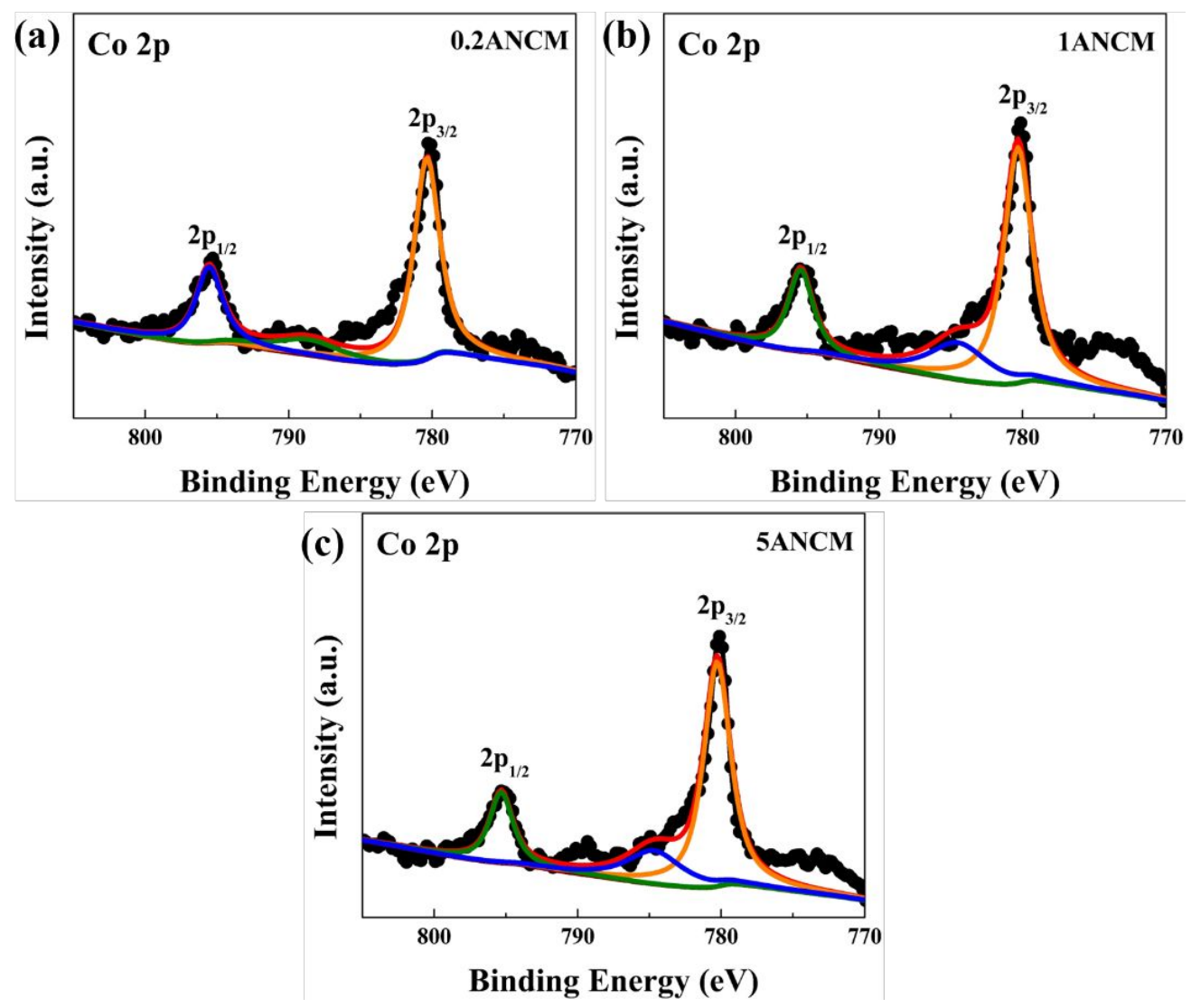

Figure S9. The high-resolution XPS spectra of Co $2 p$ for (a) 0.2ANCM, (b) 1ANCM and (c) 5ANCM cathode samples.

Table S1 Tap densities of the precursors at different reaction times $(\mathrm{g} / \mathrm{mL})$

$\begin{array}{ccccc}\text { Precursor } & 3 \mathrm{~h} & 6 \mathrm{~h} & 9 \mathrm{~h} & \mathbf{1 2 h} \\ \text { VNCM } & \mathbf{0 . 6 1} & \mathbf{0 . 9 5} & 1.40 & 1.69 \\ \text { 0.2ANCM } & \mathbf{0 . 8 7} & 1.19 & 1.40 & 1.51 \\ \text { 1ANCM } & \mathbf{0 . 7 1} & \mathbf{0 . 9 5} & 1.05 & 1.07 \\ \text { 5ANCM } & 0.69 & 0.71 & 0.79 & \mathbf{0 . 9 0}\end{array}$


Table S2 Results of different Al concentrations and the corresponding effects

\begin{tabular}{|c|c|c|c|c|c|c|}
\hline \multirow[t]{2}{*}{ Effects } & \multirow{2}{*}{$\underset{(\mathbf{a t} \%)}{\mathrm{Al}}$} & \multicolumn{2}{|c|}{ Tap Density $(\mathrm{g} / \mathrm{mL})$} & \multirow{2}{*}{$\begin{array}{c}\text { Specific } \\
\text { Surface Area } \\
\left(\mathrm{m}^{2} / \mathrm{g}\right)\end{array}$} & \multirow{2}{*}{$\begin{array}{c}\text { Specific } \\
\text { Capacity } \\
(\mathrm{mAh} / \mathrm{g})\end{array}$} & \multirow{2}{*}{$\begin{array}{c}\text { Retention } \\
\text { Capacity } \\
(\mathrm{mAh} / \mathrm{g}, 2 \mathrm{C})\end{array}$} \\
\hline & & Precursor & Cathode & & & \\
\hline VNCM & $\mathbf{0}$ & 1.69 & 2.25 & 2.215 & 179.9 & 133.4 \\
\hline 0.2ANCM & 0.25 & 1.51 & 2.08 & 3.586 & 180.2 & 138.5 \\
\hline 1ANCM & 0.96 & 1.07 & 1.83 & 2.726 & 175.7 & 119.1 \\
\hline 5ANCM & 4.77 & 0.90 & 1.73 & 3.982 & 141.6 & 88.1 \\
\hline
\end{tabular}

